\title{
Microwave Breast Imaging by the Filtered Forward- Backward Time-Stepping Method
}

\author{
Takashi Takenaka $^{\# 1}$, Toshifumi Moriyama ${ }^{\# 2}$, Kismet Anak Hong Ping ${ }^{* 3}$, Takafumi Yamasaki ${ }^{\# 4}$ \\ \# Department of Electrical and Electronic Engineering, Nagasaki University \\ 1-14 Bunkyo-machi, Nagasaki 852-8521, JAPAN \\ ${ }^{1}$ takenaka@nagasaki-u.ac.jp, ${ }^{2}$ t-moriya@nagasaki-u.ac.jp \\ ${ }^{4}$ bun.t.teufel@hotmail.co.jp \\ * Department of Electronic Engineering, Universiti Malaysia Sarawak \\ 94300 Kota Samarahan, Sarawak, Malaysia \\ hpkismet@feng.unimas.my
}

\begin{abstract}
In this paper, an inverse scattering technique referred to as the filtered forward-backward time-stepping method is applied to microwave imaging for breast cancer detection. A two-dimensional numerical breast phantom (derived from MR images) with high contrast between fat and fibroglandular tissues, and low contrast between fibroglandular and tumor tissues are used to assess the efficacy of the proposed method.
\end{abstract}

\section{INTRODUCTION}

Breast cancer is the leading cause of death from cancer among women. To reduce the breast cancer mortality rate, it is recommended for women to have annual breast screening. At present, X-ray mammography is regarded as the most effective examination modality in mass screening. However, it is less effective for detecting a tumor in a dense breast. A complementary and alternative modality has been looking for to overcome its shortcoming [1].

Over the last two decades, a number of researchers have been working on the early detection of breast cancer by microwave imaging. The microwave breast imaging techniques are classified into two approaches: one is based on the principle of radar and another is based on inverse scattering. The former images spatial distribution of the scattering intensity due to abnormalities, while the latter images spatial distribution of dielectric properties of a region of interest. The radar-based techniques get an image quickly, but only the location and size of the abnormality can be found from the image [2]-[5]. The inverse scattering-based techniques require a long processing time to get an image, but the dielectric properties of the abnormality can be obtained as well as its location and size [6]-[10]. Therefore, the imaging based on the inverse scattering techniques has a potential for tissue characterization.

Both the frequency-domain and time-domain inverse scattering techniques have been applied to the microwave breast imaging. Although the use of frequency diversity does not increase information content about a target object, it reduces ill-conditioning of the reconstruction process, so that a solution algorithm with multifrequency data becomes more stable than the use of monochromatic data [11]. We have proposed an inversion technique referred to as Forward-
Backward Time-Stepping (FBTS) algorithm [12] and applied it to breast cancer detection in two-dimensional (2-D) and three-dimensional (3-D) cases [13, 14].

Recently, the permittivity and conductivity of fibroglandular breast tissue is shown to be much higher than previously imagined [15 ]. This makes microwave imaging of a dense breast more challenging.

In this paper, the filtered FBTS technique [16] which has been shown to be more robust to noise is applied to the cancer detection of a dense breast with high-contrast between fat and fibroglandular tissues, and low-contrast between fibroglandular and tumor tissues.

\section{INVERSE SCATTERING TECHNIQUES}

We consider inverse scattering problem where the electrical property distributions within a target object are estimated from scattering time domain data. The object is surrounded by an array of antennas. Each antenna takes turns to transmit a microwave pulse while the rest of antennas collect scattered signals. Then, a set of transmitter/receiver data for multiple antenna combination is obtained.

\section{A. FBTS Inversion Algorithm}

In the FBTS technique, the measured scattering dataset is compared to an equivalent simulation in which the same set of scattering data is computed for assumed electrical property distributions within the target object. Reconstruction procedure of FBTS minimizes iteratively the following cost functional:

$$
Q(\mathbf{p})=\int_{0}^{T} \sum_{m=1}^{M} \sum_{n=1}^{N} K_{m n}(t)\left|\mathbf{v}_{m}\left(\mathbf{p} ; \mathbf{r}_{n}, t\right)-\tilde{\mathbf{v}}_{m}\left(\mathbf{r}_{n}, t\right)\right|^{2} d t
$$

where $\tilde{\mathbf{v}}_{m}\left(\mathbf{r}_{n}, t\right)$ and $\mathbf{v}_{m}\left(\mathbf{p} ; \mathbf{r}_{n}, t\right)$ are the measured electromagnetic fields in time domain at the receiver position $\mathbf{r}_{n}$ due to a transmitter $m$ and the corresponding calculated electromagnetic fields for an assumed set of electric parameters $\mathbf{p}$, respectively. The set of parameters $\mathbf{p}$ consists of the relative permittivity $\mathcal{E}_{r}$ and the conductivity $\sigma$.The factor $K_{m n}(t)$ is a nonnegative weighting function which takes a 
value of zero at time $t=T$, where $T$ is a time duration of the measurement.

When a gradient-based optimization method is applied to minimization of the cost functional (1), the gradient of the functional is necessary. By taking the Fréchet derivative of (1), the gradients with respect to $\varepsilon_{r}$ and $\sigma$ are derived as

$$
\begin{aligned}
& g_{\varepsilon_{r}}(\mathbf{r})=\int_{0}^{T} \sum_{m=1}^{M} \sum_{i=1}^{3} w_{m i}(\mathbf{p} ; \mathbf{r}, t) \frac{d}{d t} v_{m i}(\mathbf{p} ; \mathbf{r}, t) d t \\
& g_{\sigma}(\mathbf{r})=\int_{0}^{T} \sum_{m=1}^{M} \sum_{i=1}^{3} w_{m i}(\mathbf{p} ; \mathbf{r}, t) v_{m i}(\mathbf{p} ; \mathbf{r}, t) d t
\end{aligned}
$$

where $v_{m i}(\mathbf{p} ; \mathbf{r}, t)$ and $w_{m i}(\mathbf{p} ; \mathbf{r}, t)$ are the $i$ th component of the electromagnetic fields $\mathbf{v}_{m}(\mathbf{p} ; \mathbf{r}, t)$ and the adjoint fields $\mathbf{w}_{m}(\mathbf{p} ; \mathbf{r}, t)$ calculated in the reconstruction region. The adjoint fields $\mathbf{w}_{m}(\mathbf{p} ; \mathbf{r}, t)$ are time reversed fields with equivalent current sources which are identical to a difference between the measured and calculated scattered field data. In this paper, we use Polak-Ribiète-Polyak conjugate gradient method for optimization technique to solve the inverse scattering problem. The detail of the FBTS is described elsewhere [12].

\section{B. Filtered FBTS Inversion Algorithm}

A gradient-based minimization technique does not always find a global minimum of the cost functional due to nonlinearity of the inverse problem. To avoid a local minimum, a proper initial guess is essential. The use of lower frequencies reduces nonlinearity of the inverse problem. Based on the fact, frequency hopping techniques were proposed in frequency domain [11], [17].

The similar idea as frequency hopping techniques is incorporated into FBTS algorithm. Instead of multifrequency approach in frequency domain, a UWB pulse and several lowpass filters with different cutoff frequencies are used in FBTS. First, the high frequency component is removed from the measured scattered signal $\tilde{\mathbf{v}}_{m}\left(\mathbf{r}_{n}, t\right)$ by a lowpass filter with the lowest cutoff frequency. The corresponding calculated scattered signal $\mathbf{v}_{m}\left(\mathbf{p} ; \mathbf{r}_{n}, t\right)$ is also filtered by the same lowpass filter. Then, in replace of the cost functional given by (1), the following cost functional is minimized:

$$
Q(\mathbf{p})=\int_{0}^{T} \sum_{m=1}^{M} \sum_{n=1}^{N} K_{m n}(t)\left|\mathbf{V}_{m}\left(\mathbf{p} ; \mathbf{r}_{n}, t\right)-\tilde{\mathbf{V}}_{m}\left(\mathbf{r}_{n}, t\right)\right|^{2} d t
$$

where $\tilde{\mathbf{V}}_{m}\left(\mathbf{r}_{n}, t\right)$ and $\mathbf{V}_{m}\left(\mathbf{p} ; \mathbf{r}_{n}, t\right)$ are the filtered version of $\tilde{\mathbf{v}}_{m}\left(\mathbf{r}_{n}, t\right)$ and $\mathbf{v}_{m}\left(\mathbf{p} ; \mathbf{r}_{n}, t\right)$, respectively. Minimization of the cost functional (2) yields a rough image of the electrical parameter profiles $\mathbf{p}$. This reconstructed image is used as an initial guess for the filtered FBTS algorithm with a lowpass filter of the next higher cutoff frequency. This procedure is repeated successively until all the frequency components of the measured scattered signal are used (see [16] for details).

\section{NUMERICAL RECONSTRUCTION}

To assess the validity of the filtered FBTS for imaging of a breast, we examine reconstruction of a 2-D numerical breast model.

\section{A. 2-D MRI-derived Breast Model}

A slice of $0.39 \mathrm{~mm} \times 0.39 \mathrm{~mm}$ resolution MR images of the left breast of a healthy volunteer in the prone position taken at Nagasaki University Hospital was used to make a microwave 2-D breast model. First order Debye model parameters were mapped to each pixel value using the piecewise-linear mapping procedure described in [18]. The model was resized to a resolution of $1 \mathrm{~mm} \times 1 \mathrm{~mm}$, and $2 \mathrm{~mm}$ thick skin layer was added. A $5 \mathrm{~mm}$ diameter tumor was added inside the fibroglandular region. Nominal Debye parameters $\left(\varepsilon_{s}, \varepsilon_{\infty}, \sigma_{s}, \tau\right)$ for the breast model used in this paper are similar to those of [15] and summarized in Table 1. These parameters are varied linearly by $\pm 10 \%$ within each tissue region (with $\tau$ left constant). Since reconstruction of dispersionless media is considered in this work, the relative permittivity and conductivity for nominal Debye parameters evaluated at a frequency of $2.0 \mathrm{GHz}$ are as follows: fibroglandular $\varepsilon_{r}=46.95 \sigma=1.21$; fat $\varepsilon_{r}=4.69 \sigma=0.07$; skin $\varepsilon_{r}=36.73 \sigma=1.43$; tumor $\varepsilon_{r}=53.9 \sigma=1.46$. The resulting spatial distributions of the permittivity and conductivity are shown in Fig. 1(a) and (b), respectively.

TABLE I

ELECTRICAL PROPERTY PARAMETERS UTILIZED FOR BREAST MODEL.

\begin{tabular}{|l|l|c|c|c|}
\hline Tissue & $\varepsilon_{s}$ & $\mathcal{E}_{\infty}$ & $\sigma_{s}$ & $\tau$ \\
\hline fibroglandular & 47.62 & 7.04 & 0.64 & $10.3 \mathrm{e}-12$ \\
\hline fat & 4.73 & 3.14 & 0.04 & $13.6 \mathrm{e}-12$ \\
\hline skin & 37.00 & 4.00 & 1.10 & $7.2 \mathrm{e}-12$ \\
\hline tumor & 54.66 & 6.75 & 0.79 & $10.1 \mathrm{e}-12$ \\
\hline
\end{tabular}

Relative permittivity

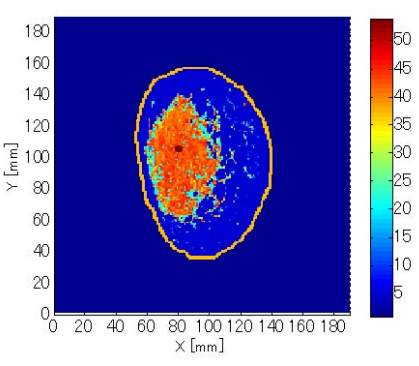

(a)
Conductivity

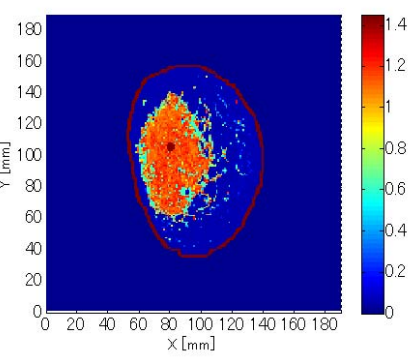

(b)
Fig. 1. A numerical breast model derived from MR images. (a) Rrelative permittivity. (b) Conductivity.

\section{B. Reconstruction}

Two-dimensional FDTD $\left(\mathrm{TM}_{\mathrm{Z}}\right.$ wave) simulations were carried out to obtain synthetic scattering data as measured scattering data. 24 points used as transmitter/receiver 
positions are arranged on a measurement circle with a radius of $18 \mathrm{~cm}$. Each of the 24 points was used sequentially as a transmitter while at the remaining positions the electric field data is collected. This set of scattering calculations formed a set of numerical measured data denoted as $\tilde{\mathbf{v}}_{m}\left(\mathbf{r}_{n}, t\right)$ term of (1). An illuminating pulse is generated by a simple line current source whose time factor is given by

$$
I(t)=t_{0}\left[\frac{d}{d t}\left(\frac{t}{t_{0}}\right)^{4} e^{-t / t_{0}}\right]
$$

where $t_{0}=0.125 \mathrm{~ns}$. The breast is assumed to be immersed in free space as a background medium.

As a lowpass filter in reconstruction procedure, a Chebyshev lowpass filter is used whose amplitude response is given by

$$
H(f)=\frac{1}{\sqrt{1+\varepsilon^{2}\left[T_{n}\left(f / f_{0}\right)\right]^{2}}}
$$

where, $\varepsilon$ is a ripple factor, $f_{0}$ is a cutoff frequency. $T_{n}(x)$ is a Chebyshev polynomial of the $n$th order and is defined by

$$
T_{n}(x)=\left\{\begin{array}{cc}
\cos \left(n \cos ^{-1} x\right) & |x| \leq 1 \\
\cosh \left(n \cosh ^{-1} x\right) & |x|>1
\end{array}\right.
$$

In the following analysis, we use a Chebyshev filter of $n=4$ and $\varepsilon=0.15$.

The FDTD solution space, bounded by a fifteen-cell convolutional perfectly matched layer (CPML), consisting of $190 \times 190$ cells with cell size $\Delta x=\Delta y=1 \mathrm{~mm}$ is used. Time duration $T$ of the measurement is $1600 \Delta t$ where the time step size $\Delta t=2.32 \mathrm{ps}$. We assume the location, thickness, and the dielectric property of the skin are a priori known. Only the inside of the breast are considered to be reconstructed. So, interior structure of the breast is imaged with the filtered FBTS method. The number of unknown elements in the reconstruction region is 15574 .

The initial $\varepsilon_{r}$ and $\sigma$ values in the entire reconstruction region were set equal to the values of free space, i.e., $\varepsilon_{r}=1$ and $\sigma=0$. Four Chebyshev filters with cutoff frequencies $f_{0}=0.5,1.0,1.5$, and $2.0 \mathrm{GHz}$ are used in the filtered FBTS reconstruction algorithm. The optimization was carried out by 200 iterations in reconstruction with each filter of a different cutoff frequency. Then, another 1200 iterations reconstruction were performed using the resulting image as an initial estimate for the FBTS method without a filter, so that the total number of iterations to get the final image is 2000 .

\section{Results}

Fig. 2 shows the reconstructed images utilizing the filtered FBTS technique. Reconstructed results of the relative permittivity $\varepsilon_{r}$ and conductivity $\sigma$ after 200 iterations using the filter of the cutoff frequency $f_{0}=0.5 \mathrm{GHz}$ are shown in
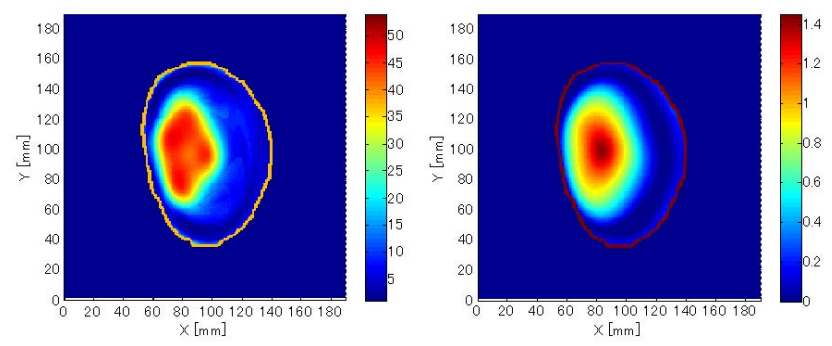

(a)
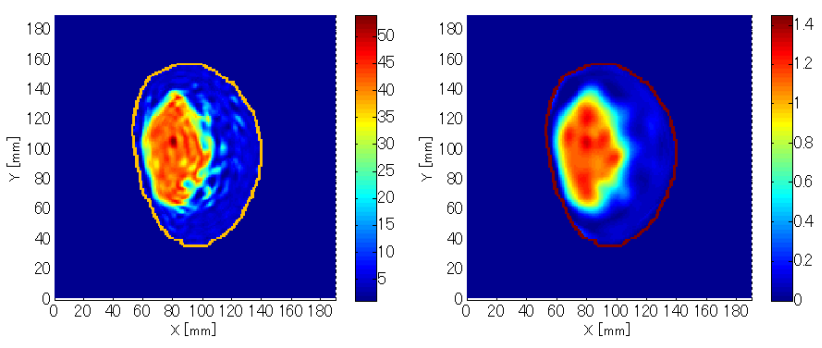

(b)
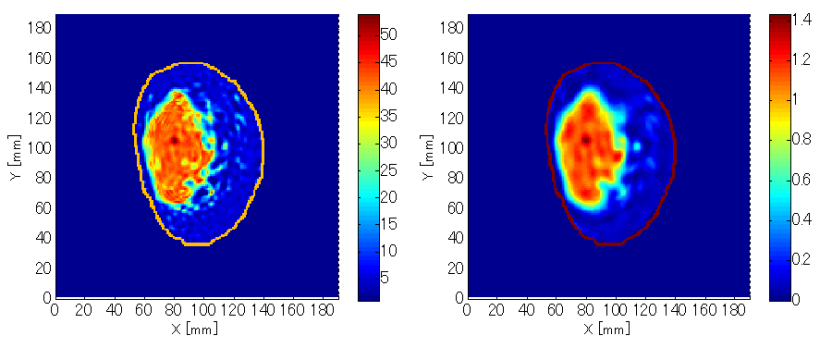

(c)

Fig. 2. Reconstruction by the filtered FBTS method with filters of cutoff frequencies $0.5,1.0,1.5$, and $2.0 \mathrm{GHz}$ followed by the FBTS method without a filter. (a) Reconstructed images after 200 iterations. (b) Reconstructed images after 1000 iterations. (c) Reconstructed images after 2000 iterations.

Fig. 2(a). The rather accurate fibroglandular region is already detected in this reconstruction. Since a large amount of frequency components of the scattered signals are lost by the filtering, it is found that the low frequency component contributes to this imaging. The reconstructed results $\varepsilon_{r}$ and $\sigma$ after 1000 iterations is shown in Fig. 2(b). The coordinates of the cell with the highest values of both reconstructed images (the relative permittivity and conductivity) are identical to the center ( $x=80 \mathrm{~mm}, y=105 \mathrm{~mm}$ ) of the tumor of the original breast model. The tumor can be clearly seen in the relative permittivity image of this figure, while unclear in the conductivity image. Fig. 2(c) shows the reconstructed results $\varepsilon_{r}$ and $\sigma$ after 2000 iterations. The conductivity image now distinctly shows the presence of a tumor in the breast. Fig. 3 shows $1-\mathrm{D}$ cuts through the 2-D permittivity and conductivity 
images of Fig. 2(c) along the vertical line cutting $x=80 \mathrm{~mm}$ through the center of the tumor. The size of the detected tumor as well as its location is quite accurate. The peak values for the tumor are $\varepsilon_{\max }=56.4$ and $\sigma_{\max }=1.43$, which is quite similar to the actual values of $\varepsilon_{\max }=53.9$ and $\sigma_{\max }=1.46$.
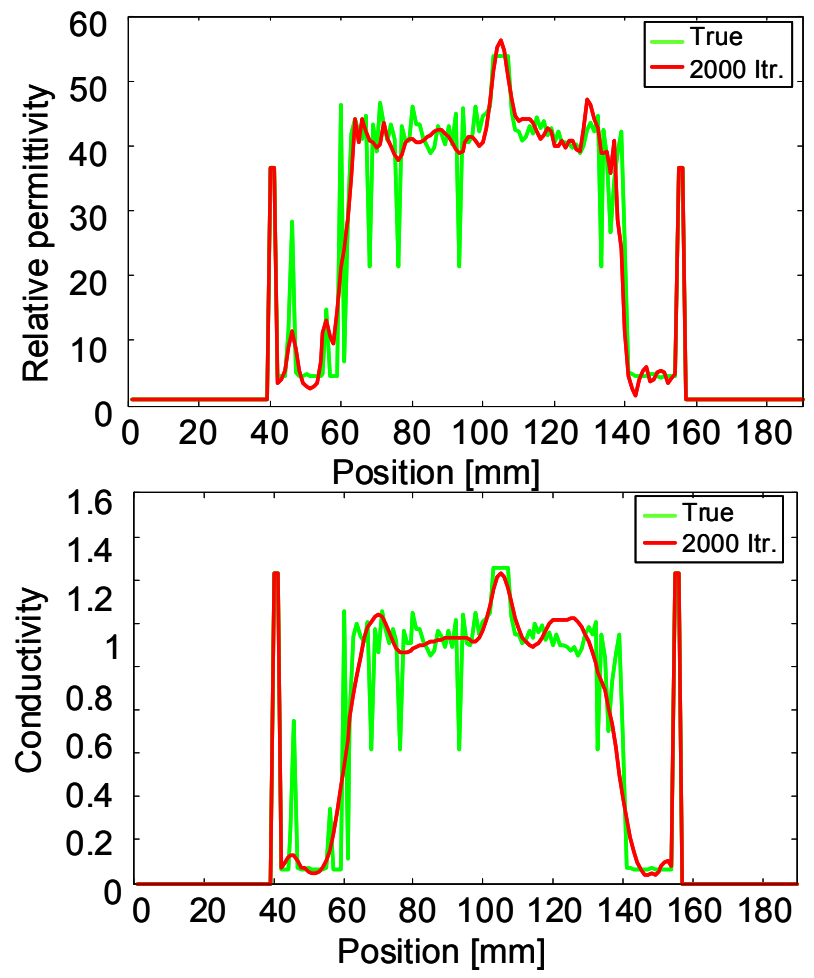

Fig. 3. 1-D cuts of Fig.2(c) along the vertical line through the center of the tumor.

\section{CONCLUSION}

The filtered FBTS inversion algorithm has been applied to imaging the interior of a breast. An MR image-based 2-D breast model was used to evaluate the inversion algorithm. The breast model has relatively higher permittivity and conductivity fibroglandular region based on a newly published work [15]. Reconstructed images indicate clearly the value of the dielectric parameters almost identical to the true values of a $5 \mathrm{~mm}$ diameter tumor placed within the fibroglandular region as well as its position and size. The numerical simulations demonstrated the utility of the proposed inverse scattering technique for detection of tumors in a dense breast.

\section{ACKNOWLEDGMENT}

This work was partially supported by a Japan Society for the Promotion of Science (JSPS) Grant-in-Aid for Scientific Research (B) (no. 20360187). The authors would like to express their thanks to Dr. Jessi Ernest Johnson for his assistance in providing the numerical data of the MRI-derived breast model.

\section{REFERENCES}

[1] Committee on Technologies for the Early Detection of Breast Cancer, Mammography and Beyond: Developing Technologies for the Early Detection of Breast Cancer, S. Nass, I. Henderson, and J. Lashof, Eds. Washington, DC: Nat. Cancer Policy Board, Inst. Med., Commission Life Stud, Nat. Res. Council, 2001.

[2] E. Bond, X. Li, S. Hagness, and B. Van Veen, "Microwave imaging via space-time beamforming for early detection of breast cancer," IEEE Trans. Antennas Propag., vol. 51, no. 8, pp. 1690-1705, Aug. 2003

[3] E. Fear, J. Sill, and M. Stuchly, "Experimental feasibility study of confocal microwave imaging for breast tumor detection," IEEE Trans. Microw. Theory Tech., vol. 51, no. 3, pp. 887-892, Mar. 2003.

[4] Y. Xie, B. Guo, L. Xu, J. Li, and P. Stoica, "Multistatic adaptive microwave imaging for early breast cancer detection," IEEE Trans. Biomed. Eng., vol. 53, pp. 1647-1657, Aug. 2006.

[5] M. Klemm, I. J. Craddock, J. Leendertz, A. Preece, and R. Benjamin, "Radar-based breast cancer detection using a hemispherical antenna array-experimental results," IEEE Trans. Antennas Propag., vol. 57, no. 6, pp. 1692-1704, Jun. 2009.

[6] S. Caorsi, A. Massa, M. Pastorino, and A. Rosani, "Microwave medical imaging: potentialities and limitations of a stochastic optimization technique," IEEE Trans. Microwave Theory Tech., vol. 52, no.8, pp. 1909-1916, Aug.2004.

[7] P. Meaney, M. Fanning, D. Li, S. Poplack, and K. Paulsen, "A clinical prototype for active microwave imaging of the breast," IEEE Trans. Microw. Theory Tech., vol. 54, no. 4, Part 2, pp. 1921-1927, Apr. 2006.

[8] A. Fhager, P. Hashemzadeh, and M. Persson, "Reconstruction quality and spectral content of an electromagnetic time-domain inversion algorithm," IEEE Trans. Biomed. Eng., vol. 53, no. 8, pp. 1594-1604, Aug. 2006.

[9] N. Irishina, M. Moscoso and O. Dorn, "Microwave imaging for early breast cancer detection using a shape-based strategy," IEEE Trans. Biomed. Eng., vol. 56, no. 4, pp. 1143-1153, Apr. 2009.

[10] D. W. Winters, J. D. Shea, P. Kosmas, B. D. Van Veen, and S. C. Hagness, "Three-dimensional microwave breast cancer imaging: dispersive dielectric properties estimation using patient-specific basis functions," IEEE Trans.Med. Imag., vol. 28, no. 7, pp. 969-981, Jul. 2009.

[11] O.M. Bucci, T. Isernia, L. Crocco, and V. Pascazio, "Inverse scattering problems with multifrequency data: reconstruction capabilities and solution strategies," IEEE Trans. Geosci. Remote Sensing, , vol. 38, no. 4, part 1, pp. 1749-1756, Jul. 2000.

[12] T. Takenaka, H. Jia, and T. Tanaka, "Microwave imaging of electrical property distributions by a forward-backward time-stepping method," J. Electromagn. Waves Appl., vol. 14, no. 12, pp. 1609-1626, 2000.

[13] J. Johnson, T. Takenaka, and T. Tanaka, "Two-dimensional timedomain inverse scattering for quantitative analysis of breast composition," IEEE Trans. Biomed. Eng., vol. 55, no. 8, pp. 19411945, Aug. 2008.

[14] J. Johnson, T. Takenaka, K. A. Hong Ping, S. Honda, and T. Tanaka, "Advances in the 3-D forward-backward time-stepping (FBTS) inverse scattering technique for breast cancer detection," IEEE Trans. Biomed. Eng., vol. 56, no. 9, pp. 2232-2243, Sep. 2009.

[15] M.Lazebnik, D. Popovic, L. McCartney, C.Watkins, M. Lindstrom, J.Harter, S. Sewall, T. Ogilvie, A. Magliocco, T. Breslin et al., "A large-scale study of the ultrawideband microwave dielectric properties of normal, benign and malignant breast tissues obtained from cancer surgeries," Phys. Med. Biol., vol. 52, no. 20, pp. 6093-6115, 2007.

[16] T. Tanaka, N. Kuroki, and T. Takenaka, "Filtered forward-backward time-stepping method applied to reconstruction of dielectric cylinders," Electromagn. Waves Appl., vol. 17, no. 2, pp. 253-270, Feb. 2003.

[17] W. C. Chew and J. H. Lin, "A frequency-hopping approach for microwave imaging of large inhomogeneous bodies," IEEE Microwave Guided Wave Lett., vol. 5, no. 12, pp. 439-441, Dec 1995.

[18] M. Converse, E. Bond, B. Veen, and S. Hagness, "A computational study of ultra-wideband versus narrowband microwave hyperthermia for breast cancer treatment," IEEE Trans. Microw. Theory Tech., vol. 54, no. 5, pp 2169-2180, May 2006. 